\title{
No reliable evidence for the association between dental crowding and caries
}

\author{
Abstracted from
}

Hafez HS, Shaarawy SM, AI-Sakiti AA, Mostafa YA.

Dental crowding as a caries risk factor: a systematic review. Am J Orthod Dentofacial Orthop 2012; 142: 443-450.

Address for correspondence: Yehya Ahmed Mostafa, Department of Orthodontics,

Oral and Dental Research Division, National Research Center, Giza, Egypt. E-mail: mangoury@usa.net

\section{Question: Is there a relationship between dental crowding and caries?}

Data sources The Cochrane Library, PubMed, Medline, LILACS (Google Scholar and ISI Web of Science databases were searched without restriction. The Journal of Dental Research, Community Dentistry and Oral Epidemiology, American Journal of Orthodontics, American Journal of Orthodontics and Dentofacial Orthopedics, Angle Orthodontist, and European Journal of Orthodontics were hand searched.

Study selection Studies in humans reporting on the prevalence or severity of dental crowding and dental caries that included a comparator or control group with no or minimal crowding and assessing the association were included. Non-English articles were excluded from the review in the study-selection stage.

Data extraction and synthesis Data extraction and evaluation of primary studies were performed independently by two reviewers. Study quality was assessed but meta-analysis was not carried out owing to the wide range of measurement indices used and study heterogeneity. A qualitative summary is presented.

Results Eight cross-sectional studies were included in the qualitative review. No association between crowding and caries was reported in four studies, a significant negative correlation was found in two studies, one study showed a direct and significant relationship and another study showed a positive association in the mandibular anterior region but an inverse correlation in the maxillary posterior region.

Conclusions To date there are no high-quality studies to resolve the possible association between dental crowding and caries; further high-quality longitudinal studies are needed to clarify this relationship.

\section{Commentary}

Since dental crowding can cause food accumulation and plaque retention, it is conceivable that dental crowding would increase the risk of dental caries. Although the association between dental crowding and caries has been studied for decades, ${ }^{1}$ a definitive conclusion has yet to be reached. This systematic review aimed to examine the association between dental crowding and caries. The authors conducted extensive electronic searching and handsearching to identify observational studies which compared the incidences of caries among subjects with no crowding or crowding of various degrees.

Eight cross-sectional studies were included and, due to great heterogeneities across studies, no meta-analysis was performed.
No association was reported in four studies, a negative correlation in two studies, a positive association in one study, and a positive association in the mandibular anterior region but an inverse correlation in the maxillary posterior region in one study. The overall quality of the included studies was not high, with seven being assessed as moderate quality and one as low quality. The included studies in this systematic review employed different criteria and indices for dental crowding. Although most of the studies used consistent indices-DMFS or DMFT—-to assess caries, these indices may underestimate the incidence of interproximal caries. Baseline oral health data were unavailable in most of the included studies, which would make the evaluation of their association more difficult. Considering the multifactorial nature of caries, it is thoughtful of the authors to include confounding factors in quality assessment. However, limited by the available data from the included studies, an integration of these factors in the interpretation of their association would be difficult. Moreover, the ages of subjects in included studies differed significantly, from children with primary dentition to adults in their 30s. Since time is needed in the development of caries, the association between dental crowding and caries may be underestimated in children or overestimated in adults with old age. Although the association was stratified into anterior and posterior segment in some of the included studies, it was not done in all the included studies.

Therefore, these aforementioned inconsistencies and heterogeneities across studies prevented the authors from drawing a definitive conclusion. Future studies are needed to clarify this association with longitudinal study design, consistent and reliable indices, comparable subjects, prolonged follow-up (preferably from children to adults), and well-balanced potential confounding factors.

\section{Practice point}

- There is no reliable evidence for the association between dental crowding and caries.

Hu Long and Wenli Lai

Department of Orthodontics, State Key Laboratory of Oral Diseases, West China Hospital of Stomatology, Sichuan University, China.

1. Collins HB. Caries and crowding in the teeth of the living Alaskan Eskimo. Am J Phys Anthropol 1932; 16: 451-462.

Evidence-Based Dentistry (2013) 14, 12. doi:10.1038/sj.ebd.6400912 